\begin{tabular}{|l|l|l|}
\hline \multicolumn{2}{|c|}{ PublisherInfo } \\
\hline \hline PublisherName & $:$ & BioMed Central \\
\hline \hline PublisherLocation & $:$ & London \\
\hline \hline PublisherImprintName & $:$ & BioMed Central \\
\hline \hline
\end{tabular}

\title{
Idoxifene antagonises E2-dependent breast cancer xenograft growth through apoptosis
}

\begin{tabular}{|l|l|l||}
\hline \multicolumn{2}{|c||}{ ArticleInfo } \\
\hline \hline ArticleID & $:$ & 3631 \\
\hline \hline ArticleDOI & $:$ & $10.1186 /$ bcr-1999-66609 \\
\hline \hline ArticleCitationID & $:$ & 66609 \\
\hline \hline ArticleSequenceNumber & $:$ & 51 \\
\hline \hline ArticleCategory & $:$ & Paper Report \\
\hline \hline ArticleFirstPage & $:$ & 1 \\
\hline \hline ArticleLastPage & $:$ & 4 \\
\hline \hline & & RegistrationDate : 1999-9-8 \\
ArticleHistory & $:$ & OnlineDate $\quad$ 1999-9-8 \\
\hline \hline ArticleCopyright & $:$ & Current Science Ltd1999 \\
\hline \hline ArticleGrants & $:$ & \\
\hline \hline ArticleContext & $:$ & 1305811 \\
\hline \hline
\end{tabular}




\section{Keywords}

Apoptosis, idoxifene, MCF-7 xenograft

\section{Introduction}

Idoxifene is an analogue of tamoxifen currently being developed for the treatment of breast cancer. In comparison to tamoxifen, idoxifene is metabolically more stable and demonstrates a higher relative binding affinity for the estrogen receptor (ER). As a result, idoxifene is more effective in inhibiting tumour cell growth in both in vitro and in vivo models. Idoxifene also inhibits calmodulin, a calciumbinding protein that is involved in cell signal transduction pathways. The formation of calmodulin-ER complexes is thought to be a key step in tumour growth and it has been shown that the cytotoxicity of tamoxifen analogues correlates with their calmodulin antagonism. The cis isomer of idoxifene has a lower binding affinity for ER than the clinically used transisomer, but it still has potent calmodulin antagonism. This difference between the two isomers opens up the possibility of examining which of the two properties (ER antagonism and calmodulin antagonism) is more important in inhibiting tumour growth. Finally, idoxifene demonstrates reduced agonist activity on breast and uterine cells.

\section{Aims}

The aims of this study were twofold. The first aim was to compare the abilities of idoxifene and tamoxifen to antagonize estrogen (E2)-dependent MCF-7 xenograft growth in oophorectomized athymic mice. This was examined in relation to the ability of the drugs to antagonize ER and to antagonize the formation of calmodulin-ER complexes. The second aim was to compare the effects of idoxifene, its cisisomer and tamoxifen on proliferation and apoptosis in the harvested xenograft tumours.

\section{Comments}

This is a well conducted study demonstrating the similar effects of idoxifene and tamoxifen on inhibiting E2-dependent xenograft breast tumour growth. Most interesting were the biological marker studies showing that, while both drugs caused an early increase in apoptosis, only idoxifene caused a prolonged effect. It would naturally be of interest to see if this effect could be maintained for longer 
periods or whether this too would eventually end. If the effect can be maintained, then it suggests the possibility that idoxifene, as compared to tamoxifen, may lead to longer periods of tumour control.

\section{Methods}

The three drugs [tamoxifen, trans idoxifene (referred to as Idoxifene), and cis idoxifene] were administered to the mice via subcutaneous implantation of slow-release silastic capsules. Ninety five randomly bred female athymic mice were oophorectomized. Following recovery, MCF-7 tumour was implanted under the mammary fat pad of each mouse and then growth-stimulated by a slow release E2 pellet. After four weeks, when tumours had grown sufficiently, 90 mice were divided into five treatment groups: E2 plus placebo; E2 plus tamoxifen; E2 plus idoxifene; E2 plus cisidoxifene; and withdrawal of E2 support and placebo. Five mice from each group were sacrificed at one week and the rest were sacrificed at 12 weeks. Tumour volumes were measured weekly. Changes in tumour cell proliferation and apoptosis (measured immunohistochemically) and ER-dependent protein expression were studied.

\section{Results}

Both idoxifene and tamoxifen significantly inhibited E2-dependent tumour growth (no significant difference between the two), whereas cis idoxifene had little effect. Withdrawal of E2 led to almost complete regression of tumours.

Uterine weights: Animals treated with E2 plus tamoxifen or idoxifene had significantly lower uterine weights than those treated with E2 alone

Cell proliferation: There were decreased proliferation rates (as measured by mean Ki-67 score) caused by both tamoxifen and idoxifene, although this effect appeared to disappear after three months of treatment (controls) but decreased back to baseline in tumours treated with tamoxifen (0.69\%). In contrast, cis idoxifene had no effect on cell proliferation.

Apoptosis index: Both anti-E2s caused a significant threefold induction of apoptosis in E2 supported tumours after one week, which was maintained for three months with idoxifene (3.1 versus $0.48 \%$ compared to E2 controls) but decreased back to baseline in tumours treated with tamoxifen $(0.69 \%)$. In contrast, cis idoxifene had no effect on apoptosis.

ER and progesterone receptor (PgR):Both tamoxifen and idoxifene initially induced ER expression. Prolonged therapy with tamoxifen significantly reduced progesterone receptor levels.

\section{Discussion}


Both idoxifene and tamoxifen caused similar inhibition of E2-dependent MCF-7 xenograft growth. Since the cis isomer of idoxifene had little impact on tumour growth, this suggests that the antitumour effect is mediated via ER rather than through calmodulin. There is no evidence that idoxifene is converted into its cis isomer in vivo. Tamoxifen and idoxifene have similar antagonistic impact on the effect of E2 on uterine tissue. Previous work has shown that idoxifene has significantly less agonist activity on uterine tissue than does tamoxifen. Sustained induction of apoptosis may contribute to prolonged antagonism of E2-dependent growth, and it occurred to a greater extentfollowing three months of idoxifene, compared to tamoxifen.

\section{References}

1. Johnston SRD, Boeddinghaus IM, Riddler S, Haynes BP, Hardcastle IR, Rowlands M, Grimshaw R, Jarman M, Dowsett M: Idoxifene antagonizes estradiol-dependent MCF-7 breast cancer xenograft growth. Cancer Res. 1999, 59: 3646-3651. 